\title{
Opioid Misuse in Missouri: Analyzing Emergency Department Use in Urban/Rural Areas
}

\author{
Evan Mobley*, Andrew Hunter and Whitney Coffey \\ Bureau of Health Care Analysis and Data Dissemination, Missouri Department of Health and Senior Services, Jefferson City, MO, USA
}

\section{Objective}

Compare rate changes over time for Emergency Department (ED) visits due to opioid overdose in urban versus rural areas of the state of Missouri.

\section{Introduction}

Like many other states in the U.S., Missouri has experienced large increases in opioid abuse resulting in hundreds dying each year and thousands of ED visits due to overdose. Missouri has two major urban areas, St. Louis and Kansas City and a few smaller cities, while the remainder of the state is more rural in nature. The opioid epidemic has impacted all areas in the state but the magnitude of that impact varies as well as the type of opioid used. Missouri Department of Health and Senior Services (MODHSS) maintains the Patient Abstract System (PAS) which contains data from hospitals and ambulatory surgical centers throughout the state. PAS includes data from ED visits including information on diagnoses, patient demographics, and other information about the visit. MODHSS also participates in the Enhanced State Surveillance of Opioid-involved Morbidity and Mortality project (ESOOS). One major aspect of this surveillance project is the collection of data on non-fatal opioid overdoses from ED visits. Through this collection of data, MODHSS analyzed opioid overdose visits throughout the state, how rates compare across urban and rural areas, and how those rates have changed over time.

\section{Methods}

The 115 counties in Missouri were organized into the six-level urban-rural classification scheme developed by the National Center for Health Statistics (NCHS). The attached table shows the breakout of counties into the six different categories. The data years analyzed were 2012 through 2016. ED visits due to opioid overdose were identified using case definitions supplied by ESOOS. Overdoses were analyzed in three different categories - all opioids, heroin, and non-heroin opioids. The all opioid category combines heroin and non-heroin opioids. Non-heroin opioids includes prescription drugs such as oxycodone, hydrocodone, fentanyl, and fentanyl analogues. Annual rates per 10,000 were calculated for each county classification using population estimates. Confidence intervals (at 95\%) were then calculated using either inverse gamma when the number of ED visits was under 500, or Poisson when the number was 500 or more. Changes over time were calculated using both a year over year method and a 5 year change method.

\section{Results}

Overall opioid rates have increased in all geographic areas during the 5 year period analyzed. Large Central Metro and Large Fringe Metro counties had the highest rates of ED visits due to opioid overdose. These two classifications also saw the largest increases in rates. The Large Central Metro counties collectively increased over $125 \%$, while the Large Fringe Metro area increased $130 \%$. Both areas experienced statistically significant increases year-to-year between 2014 and 2016 in addition to the overall 5 year period of 2012-2016.

Analysis was also conducted for heroin and non-heroin subsets of opioid abuse. There were important differences in these two groups. For heroin ED visits, the highest rates were found in the Large
Central Metro and Large Fringe Metro regions. However, the largest increase in percentage terms were found in the Medium Metropolitan, Micropolitan and Noncore regions which all saw increases of over $300 \%$. Notably, every region experienced increases of over $150 \%$. The Medium Metro had two consecutive years (2013/2014 and 2014/2015) where the heroin ED rate more than doubled.

In contrast, non-heroin ED visits did not experience such a large increase over time. Most areas saw small fluctuations year-to-year with moderate overall increases over the 5 -year time period. The exception to this trend is the Large Fringe Metro area, which saw increases every year most notably between 2014 and 2015 and had by far the largest 5 year increase at $82 \%$.

\section{Conclusions}

The urban areas in Missouri continue to have the highest rates of opioid overdose, however all areas within the state have experienced very large increases in heroin ED visits within the past five years. The increase in heroin ED visits in the rural areas suggests the abuse of heroin has now spread throughout the state, as rates were much lower in 2012. The steady increase in non-heroin opioids unique to the Large Fringe Metro may be due to the availability of fentanyl in urban areas especially the St. Louis area. This possible finding would correspond with the increased deaths due to fentanyl experienced in and around the St. Louis urban area that has been identified through analysis of death certificate data.

\begin{tabular}{|c|c|c|c|c|c|}
\hline \multicolumn{6}{|c|}{ MO Resident Heroin Percent Change } \\
\hline & $2012 / 2013$ & 2013/2014 & $2014 / 2015$ & $\begin{array}{l}2015 / 2016 \\
\text { (pv) }\end{array}$ & $2012 / 2016$ \\
\hline Large Central Metro & $15 \%$ & $30 \%$ & $32 \%$ & $61 \%$ & $219 \%$ \\
\hline Large Fringe Metro & $-8 \%$ & $28 \%$ & $35 \%$ & $58 \%$ & $151 \%$ \\
\hline Medium Metro & $-21 \%$ & $223 \%$ & $178 \%$ & $-10 \%$ & $542 \%$ \\
\hline Micropolitan & $76 \%$ & $6 \%$ & $63 \%$ & $43 \%$ & $336 \%$ \\
\hline Noncore & $54 \%$ & $35 \%$ & $138 \%$ & $1 \%$ & $399 \%$ \\
\hline Small Metro & $27 \%$ & $85 \%$ & $7 \%$ & $49 \%$ & $275 \%$ \\
\hline Total & $2 \%$ & $31 \%$ & $41 \%$ & $50 \%$ & $183 \%$ \\
\hline
\end{tabular}

\begin{tabular}{|l|r|r|r|r|r|}
\hline \multicolumn{7}{|c|}{ MO Resident Non-Heroin Percent Change } \\
\hline & $2012 / 2013$ & $2013 / 2014$ & $2014 / 2015$ & $\begin{array}{l}2015 / 2016 \\
\text { (pv) }\end{array}$ & $2012 / 2016$ \\
\hline Large Central Metro & $-19 \%$ & $-8 \%$ & $30 \%$ & $31 \%$ & $26 \%$ \\
\hline Large Fringe Metro & $7 \%$ & $2 \%$ & $40 \%$ & $19 \%$ & $82 \%$ \\
\hline Medium Metro & $-13 \%$ & $-11 \%$ & $39 \%$ & $-7 \%$ & $0 \%$ \\
\hline Micropolitan & $4 \%$ & $-3 \%$ & $16 \%$ & $-2 \%$ & $15 \%$ \\
\hline Noncore & $1 \%$ & $-6 \%$ & $-1 \%$ & $-21 \%$ & $-25 \%$ \\
\hline Small Metro & $3 \%$ & $17 \%$ & $-11 \%$ & $24 \%$ & $33 \%$ \\
\hline Total & $-3 \%$ & $-2 \%$ & $21 \%$ & $10 \%$ & $26 \%$ \\
\hline
\end{tabular}

\begin{tabular}{|l|l|l|l|}
\hline Increase & $>100 \%$ & $50-100 \%$ & $<50 \%$ \\
\hline Decrease & $<50 \%$ & & \\
\hline
\end{tabular}


ISDS 2018 Conference Abstracts

\begin{tabular}{|l|r|}
\hline $\begin{array}{l}\text { County } \\
\text { Classification }\end{array}$ & $\begin{array}{l}\text { Number of } \\
\text { Counties }\end{array}$ \\
\hline Large Central Metro & 2 \\
\hline Large Fringe Metro & 14 \\
\hline Medium Metro & 6 \\
\hline Micropolitan & 22 \\
\hline Noncore & 59 \\
\hline Small Metro & 12 \\
\hline Grand Total & 115 \\
\hline
\end{tabular}

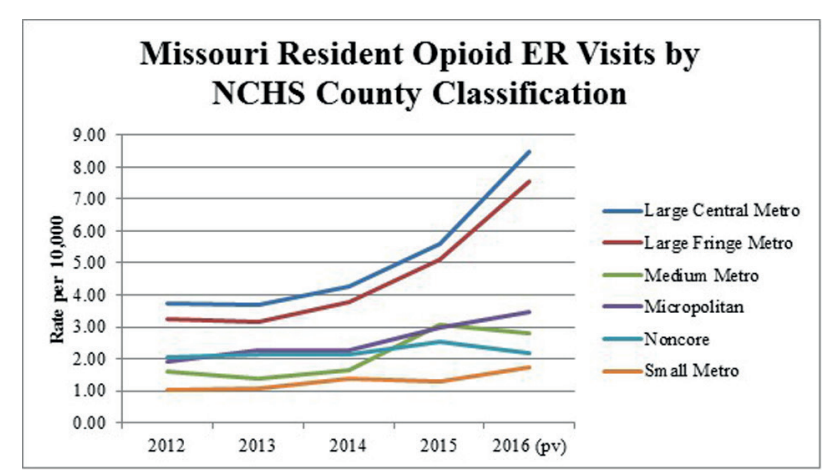

Keywords

Opioid; Urban/Rural; Emergency Department; Heroin; Fentanyl

\section{*Evan Mobley}

E-mail: Evan.Mobley@health.mo.gov 